\title{
Caspase-11, the Main Executioner in Non-canonical Inflammasome
}

\author{
Zahid Manzoor and Young-Sang Koh ${ }^{*}$ \\ Department of Microbiology and Immunology, Jeju National University School of Medicine, Jeju, Korea
}

\begin{abstract}
Murine caspase-11 is orthologus to human caspase- 4 and caspase-5, and is required for the response to cholera toxin B and infection with Escherichia coli, Citrobacter rodentium or Vibrio cholerae. Caspase-11 rather than caspase-1 is the main offender involved in sepsis. Hence, caspase-11 plays an essential pro-inflammatory role in innate immune response to bacterial infections.
\end{abstract}

Key Words: Caspase-11, Caspase-1, Sepsis, Inflammasome

In Nature on $3^{\text {rd }}$ November 2011, Kayagaki et al reported that non-canonical inflammasome activation targets caspase11 which is the main effector involved in sepsis (1). Sepsis is a potentially lethal medical condition, characterized by whole-body inflammatory state and the presence of a known infection. Interleukin-1 alpha (IL-1 $\alpha$ ), a protein of the interleukin-1 family which is secreted by activated macrophages and is responsible for inflammation, as well as the promotion of sepsis. Various classes of drugs are being developed to interrupt those processes and treat inflammatory conditions (2). Activation of inflammasome stimulates inflammation by secretion of the pro-inflammatory cytokines interleukin (IL)-1 $\beta$ and IL-18 and through release of the high-mobility group box1 (HMGB1) $(3,4)$. Inflammasome, a multi protein structure, can be activated by a variety of stimuli including some crystals, ATP and bacterial toxins (5). Salmonella or flagellin is involved in engagement of

Received: May 13, 2012/ Revised: May 17, 2012

Accepted: May 18, 2012

* Corresponding author: Young-Sang Koh. Department of Microbiology and Immunology, Jeju National University School of Medicine, 102 Jejudaehakno, Jeju 690-756, Korea.

Phone: +82-64-754-3851, Fax: +82-64-702-2687

e-mail: yskoh7@jejunu.ac.kr

** This study was supported by Basic Science Research Program through the National Research Foundation of Korea (NRF) funded by the Ministry of Education, Science and Technology, Republic of Korea (2010-0023645).
NLRC4 dependant inflammasome and double stranded DNA or Francisella tularensis activates absent in melanoma2 (AIM2) inflammasome (1, 3, 5). External ATP, mono sodium urate, silica and calcium pyrophosphate are the known activator of NLRP3 inflammasome and described as canonical stimuli $(1,5)$. Here we summarize their results regarding non-canonical inflammasome which targets caspase-11 and plays an important role in sepsis (1).

Caspase- 1 previously known as interlukin- $1 \beta$ converting enzyme (ICE) is involved in proteolytic processing and release of IL-1 $\beta$. All caspases are synthesized as inactive enzymes, termed as pro-caspases (6). Caspases are regulated at post-translational level, so they can be rapidly activated. Caspase-3 and -7 are involved in apoptotic cell death which is programmed and is not inflammatory. Activation of caspase-1 is carried out in multiprotein structure called as inflammasome. Caspase-1 is involved in another type of programmed cell death known as pyroptosis which differs from apoptosis (3). Caspase-11 is a member of caspase-1 subfamily of proteases and is most homologous to human caspase-4. The function of caspase-11 has not been fully known, but it is believed to be an inflammatory caspase, along with caspase-1 (6). Caspase- 11 has $46 \%$ similarities to caspase- 1 and is involved in secretion of IL- $\alpha$ and HMGB1 which is important inflammatory mediator. Casp1 
and Casp11 are present very close in the mouse genome, so all $\mathrm{Casp1}^{-/-}$mice that have been generated in 129 strains are also defective in Casp11 and called as Casp1/11 double knockout mice (1). Although genome of the 129 mouse strain was sequenced several years ago but now researchers realized that gene encoding caspase-11 is dysfunctional.

Cholera toxin B (CTB) which is a component of the AB5 holotoxin complex, lacks the enzymatic activity necessary for enteropathogenesis but facilitates the entry of enzymatic component cholera toxin A. By western blotting and quantitative polymerase chain reaction along with reverse transcription (RT-PCR), Kayagaki and his colleagues confirmed that Casp11 is mutated in mouse strain 129. Sequencing of 129 strain genomic DNA acknowledged a 5-bp deletion encircling the exon 7 which results in truncated Casp11. Mutation of Casp11 in mouse strain 129 abolishes the activation of inflammasome by CTB (1). To investigate the role of caspase-11 in inflammasome activation Kayagaki et al generated Casp $11^{-/-}$mice by targeting Casp11 exon 5 which contain the critical catalytic residue cysteine 254 (1). The canonical stimuli (ATP, F. tularensis, P. aeruginosa, flagellin, mono sodium urate, nigericin and calcium pyrophosphate) stimulated similar amount of IL-1 $\beta$ secretion from wild type and Casp $11^{-/-}$bone marrow-derived macrophages (BMDMs). However caspase-11 was un-dispensable for CTB, E. coli, C. rodentium and Vibrio cholerae. They called these caspase-11 dependant inflammasome as noncanonical inflammasome. In case of canonical inflammasome activator, caspase-1 is required for proteolytic processing and release of IL-1 $\beta$, IL-18, IL-1 $\alpha$, HMGB1 and pyroptosis. As long as non-canonical inflammasome stimulator (CTB, E. coli, C. rodentium and Vibrio cholerae) are concerned, activated caspase-11 results in secretion of IL-1 $\alpha$, HMGB1 and can give death signal independent of caspase-1 (1). They found that CTB and E. coli both induced secretion of caspase-11 independent of NLRP3 and ASC. The mechanism of caspase-11 activation remains unknown; however, apart from stimulator, caspase-1 is required for proteolytic processing of IL-1 $\beta$ and IL-18 (1).

Kayagaki et al generated new mice that were deficient in caspase-1, but not caspase-11 to identify the role of caspase-11. They microinjected a Casp11 bacterial artificial chromosome transgene (Tg) into Casp1/11 double knockout embryos and generated (Casp1 ${ }^{-/-}$Casp $11^{\mathrm{Tg}}$ ) mice lacking

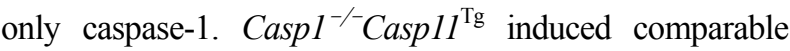
LDH release to wild type BMDMs, while Casp1/11 double knockout and Casp $11^{-1-}$ BMDMs resulted in an improved survival. This pointed out that caspase- 1 is not essential for caspase-11 activation and macrophage death stimulated by the non-canonical inflammasome (1). In case of canonical activators only caspase- 1 is required for macrophage death and release of IL-1 $\alpha(1)$.

When they injected intraperitoneally a lethal dose of LPS $(54 \mathrm{mg} / \mathrm{kg})$ which is a model of acute septic shock, they found that Casp11 ${ }^{-/-}$or Casp1/11 double knockout

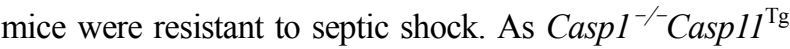
mice succumbed to infection and died like wild type mice, so it was concluded that caspase-11 instead of caspase-1 may be the main effector of LPS-induced lethal septic shock (1). To investigate the further role of caspase-11, they injected LPS $20 \mathrm{mg} / \mathrm{kg}$ intraperitoneally, and observed that Casp11 ${ }^{-1-}$, Casp1/11 double knockout or Casp1 ${ }^{-/-}$Casp11 ${ }^{\mathrm{Tg}}$ had less serum level of IL-1 $\beta$ and IL-18 after $12 \mathrm{hrs}$ as compared to wild type mice. This shows that caspase- 1 as well as caspase-11 was essential for IL-1 $\beta$ and IL-18 secretion in vivo (1). The production of IL-1 $\alpha$ which is involved in promotion of sepsis and fever, was also very less in Casp11 $11^{-/}$or Casp1/11 double knockout mice as compared to wild type mice. IL-1 receptor knockout and wild type mice showed similar response to LPS induced lethal septic shock, so the question that what causes the lethality downstream of caspase-11 is yet to be solved (1).

Caspase-11 triggers caspase- 1 dependant production of IL-1 $\beta$ and IL-18 and caspase-1 independent macrophage death in response to non-canonical activators. Kayagaki et al show that caspase-11 instead of caspase-1 may be an important effector of inflammatory response. Hence, they suggested that caspase- 4 and caspase- 5 may be more effective therapeutic targets compared to caspase- 1 in patients suffering from sepsis. 


\section{REFERENCES}

1) Kayagaki N, Warming S, Lamkanfi M, Vande Walle L, Louie S, Dong J, et al. Non-canonical inflammasome activation targets caspase-11. Nature 2011;479:117-21.

2) Dinarello CA. Induction of interleukin-1 and interleukin1 receptor antagonist. Semin Oncol 1997;24:S9-81-S9 $-93$.

3) Vande Walle L, Lamkanfi M. Inflammasomes: caspase1-activating platforms with critical roles in host defense.
Front Microbiol 2011;2:3.

4) Liu Z, Zaki MH, Vogel P, Gurung P, Finlay BB, Deng $\mathrm{W}$, et al. Role of inflammasomes in host defense against Citrobacter rodentium infection. J Biol Chem 2012;287:16955-64.

5)Hong S, Park S, Yu JW. Pyrin domain (PYD)containing inflammasome in innate immunity. J Bacteriol Virol 2011;41:133-46.

6) Wang S, Miura M, Jung YK, Zhu H, Li E, Yuan J. Murine caspase-11, an ICE-interacting protease, is essential for the activation of ICE. Cell 1998;92:501-9. 